\title{
Thermoelectric behaviour of Bi-Te films on polymer substrates DC-sputtered at room- temperature in moving web deposition
}

Xudong Tao ${ }^{a}$, Kening Wan $^{b}$, Joshua Deru ${ }^{a}$, Emiliano Bilotti ${ }^{b}$, Hazel E. Assender ${ }^{a}$.

a: Department of Materials, University of Oxford, Parks Road, OX1 3PH

b: School of Engineering and Materials Science, Queen Mary University of London, Mile End Road, E1 4NS.

Corresponding Author: Hazel E. Assender, hazel.assender@materials.ox.ac.uk

Keywords: flexible/wearable electronics, room-temperature sputtering, Bismuth Telluride, thermoelectric materials, roll-to-roll processing.

\section{Abstract}

High-throughput roll-to-roll processing could be used to scale up the manufacture of flexible thermoelectric generators. Very thin thermoelectric layers can be manufactured at high throughput speed and low cost and, most importantly, are predicted to possess better thermoelectric properties than thicker layers. Here we present a study on a series of bismuth telluride films of different thickness (few $\mathrm{nm}$ to $370 \mathrm{~nm}$ ), deposited on polymer substrates at room temperature using DC magnetron sputtering. Unlike previous studies of deposition of bismuth telluride films onto heated substrates, an island-growth mode, indicated by AFM, was observed for Bi-Te films grown at room temperature. A period of growth in which the layer only partially coats the substrate, with only imperfect connections between islands, was observed. In this partially coated region, the coating exhibited an extremely high Seebeck coefficient. An energy barrier mechanism, similar to the interface effect in nanomaterials, is proposed to explain this phenomenon, along with a possible quantum confinement effect. We found that a thinner $\mathrm{Bi}$-Te film could generate a greater power factor because of a quasidecoupling of Seebeck coefficient and electrical resistivity. In addition, ensuring that the 
sample passed directly under the sputtering target, and using a substrate smoothed with an acrylate layer were found to improve film properties, thus enhancing thermoelectric behaviour.

\section{Introduction}

Since wearable thermoelectric generators (TEGs) are of interest as a clean source of local energy [1], thermoelectric (TE) materials for near-room temperature (RT) operation have been extensively studied. Based on Seebeck effect, TE materials can directly convert thermal energy to electrical energy and vice versa [2]. As one of the best conventional TE materials at $\mathrm{RT}$, bismuth telluride $\left(\mathrm{Bi}_{2} \mathrm{Te}_{3}\right)$ is a type of $\mathrm{V}_{2}-\mathrm{VI}_{3}$ binary chalcogenide compound semiconductor with a narrow band gap $\left(E_{g}\right)$ of around $0.2 \mathrm{eV}$ [3-5]. In addition to the RT working regime, $\mathrm{Bi}_{2} \mathrm{Te}_{3}$ exhibits relatively high Seebeck coefficient, low electrical resistivity and low thermal conductivity [6]. However, the dimensionless figure of merit for TE performance (ZT) of $\mathrm{Bi}_{2} \mathrm{Te}_{3}$ has remained around 1 for many years [7]:

$$
Z T=\frac{S^{2} T}{\rho \lambda}=\frac{S^{2} \sigma T}{\lambda}=P F \cdot \frac{T}{\lambda}
$$

where $S, T, \rho, \sigma, \lambda$ and $P F$ are the Seebeck coefficient, the absolute temperature, the electrical resistivity, the electrical conductivity, the thermal conductivity, and the power factor, respectively [8]. PF is introduced because it is independent of $\lambda$. A high-efficiency TE material is required to possess low $\rho$ and high $S$, thereby high $P F$. However, $\rho, \lambda$ and $S$ are strongly coupled to the carrier concentration $[9,10]$. The main challenge remains on how to decouple these three TE parameters to maximise ZT. In recent years, nanostructured materials and boundary or defect engineering have increasingly attracted the attention of researchers for improving the $Z T$ of TE materials [11-14] because nanostructures can provide a chance to break the linkage among $\rho, \lambda$ and $S$, and thus potentially enhance the TE performance. 
Due to a poor mechanical flexibility of bulk inorganic materials, fabricating inorganic materials into thin-film form onto a flexible organic substrate is a promising option for flexible TEGs [15] . Various techniques have been reported in the literature for the fabrication of $\mathrm{Bi}_{2} \mathrm{Te}_{3}$ films, such as pulsed laser deposition $[16,17]$, molecular beam epitaxy $[18,19]$, metal organic chemical vapour deposition [20,21], electro-deposition [22], (co-)evaporation [23-28], and (co-)sputtering deposition [29-32]. The sputtering technique shows particular promise for scale-up manufacturing of TEGs: it is widely used in industrial manufacturing, being a continuous, stable and scalable process for making high quality films at moderate throughput speeds. Sputtering can be easily incorporated into roll-to-roll (R2R) manufacturing [33], as well as being compatible with fabrication lines to deposit a huge array of materials [34]. Hence, sputter deposition is employed in this study.

TEGs can be deposited onto flexible polymer substrates to give rise to good mechanical flexibility e.g. for wearable technologies [35]. Typically, the best performing materials have been achieved by deposition onto a heated substrate: $\mathrm{Bi}_{2} \mathrm{Te}_{3}$ films grow by a single crystalline 2D layer-by-layer growth mode [36-38] or a 2D step flow growth mode [39,40], which causes a columnar growth of $\mathrm{Bi}_{2} \mathrm{Te}_{3}$ thereby increasing $Z T$ [41-44]. According to the zone model proposed by Thornton in 1973 [45], a columnar-growth film can only be achieved when the ratio of the substrate temperature to the target's melting point is around 0.4 , which requires the substrate temperature to be at least $232{ }^{\circ} \mathrm{C}$ for $\mathrm{Bi}_{2} \mathrm{Te}_{3}$. However, such high temperatures, combined with the mechanical loadings applied during R2R deposition processes, can be prohibitive for most polymer substrates. Additionally, heating a polymer substrate in a highthroughput R2R process is an engineering challenge in itself. Therefore, there is an urgent desire to further study $\mathrm{Bi}_{2} \mathrm{Te}_{3}$ films grown at $\mathrm{RT}$.

$\mathrm{Bi}_{2} \mathrm{Te}_{3}$ is predicted to be a topological insulator, and has shown topological surface states that are protected by time reversal symmetry $[46,47]$. The expected conducting surface and insulating bulk would give rise to a linear decrease in $\rho$ with a decrease in thickness of a film 
[48]. A range of recent thickness studies [49-53], although not showing a linear relationship with thickness explicitly, do show an increase of resistivity with thickness, as would be expected. A lower $\rho$ benefits $P F$ (see eq. 1), but the change in $S$ with thickness is not clear for Bi-Te film sputtered at RT. Materials of very small dimensions are predicted to show significant increases in PF due to quantum confinement effects [54]. For example, Mingo et al. [55] showed an increase in PF of TE nanowires of various materials (InP, GaAs, InAs and InSb) as the wire thickness became smaller. By far the thinnest $\mathrm{Bi}_{2} \mathrm{Te}_{3}$ thin-film study, around $18 \mathrm{~nm}$ with $S \approx 100 \mu \mathrm{V} / \mathrm{K}$, was reported by Rogacheva et al. [56] using thermal evaporation onto a hot substrate as the deposition method. Such a relationship opens up the exciting possibility of improved TE properties for very thin films, which would also have the manufacturing advantage of high deposition throughputs, to keep down the manufacturing cost. Therefore, in this paper we explore the TE properties of $\mathrm{Bi}_{2} \mathrm{Te}_{3}$ thin films, particularly down to very thin layers, by RT sputter deposition onto polymer substrates.

\section{Experimental details}

$\mathrm{Bi}_{2} \mathrm{Te}_{3}$ material used in this study was supplied by Mi-Net Technology Ltd. with a purity of 99.999\%. Bi-Te films were deposited by DC magnetron sputtering onto flexible polyethylene terephthalate (PET) film $(0.1 \mathrm{~mm})$ at RT $0.25 \mathrm{~kW}$ under a vacuum of $2 \times 10^{-4} \mathrm{mbar}$ base pressure $\left(\approx 1 \times 10^{-3}\right.$ mbar after 350 argon flow, was indicated by the chamber gauge during coating) which is a typical pressure for a low-cost high throughput R2R process that we seek to emulate. At these pressures we would expect to see some effects of impurities in the film e.g. oxides, however in this study we have not modified pressure, deposition rate, power or temperature, so we would not expect major trends in the effect of such impurities. For some samples, acrylate smoothing layers were previously vacuum-deposited onto the PET film, as contrast substrates, by flash evaporation of a tripropyleneglycol diacrylate (TPGDA) monomer followed by plasma cure [57]. With the help of polyimide tapes, substrates were attached on the coating drum (circumference $1.8 \mathrm{~m}$ ), which rotates at $25 \mathrm{~m} / \mathrm{min}$ during 
deposition, and thus multiple passes of the deposition source were made during a single deposition process. The film thickness was controlled by changing the deposition time, and then measured using a Veeco DekTak $6 \mathrm{M}$ stylus profiler, which measured, in at least 5 locations, the step-height between the film and the substrate made by masking with polyimide tapes. A series of thin films with varying thickness were obtained as shown in Table I. In order to manufacture ultra-thin films, PET substrates were also placed towards the edge of the drum, i.e. not passing directly underneath the sputter target as the cathode/target is much smaller than the drum's width. This oblique deposition can produce thinner films compared to directly opposite deposition.

Table I. Bi-Te samples obtained by varying deposition time. The thickness of the thinnest layers ' ' are estimated, as they form non-continuous layers. Note that the 'deposition time' is the time that the sample is rotating on the drum during deposition: the time spent under the sputter cathode is substantially less.

\begin{tabular}{|c|c|c|c|c|c|c|c|c|}
\hline Position of target and substrate & \multicolumn{6}{|c|}{ Directly opposite deposition } & \multicolumn{3}{c|}{ Oblique deposition } \\
\hline Deposition time (mins) & 10 & 5 & 3 & 2 & 1 & 5 & 3 & 1 \\
\hline Film thickness (nm) & 370 & 170 & 100 & 70 & 20 & 55 & $\sim 33$ & $\sim 11$ \\
\hline Standard deviation (n=5) & 3 & 4 & 5 & 3 & 1 & 3 & - & - \\
\hline
\end{tabular}

AFM (JEOL JSTM-4200D) using tips (NCHV-A, Bruker Ltd.) in tapping mode, and a fieldemission scanning electron microscope (FE-SEM Zeiss Merlin) were used to analyse the film surface morphology, and to determine the surface roughness of films. $E_{g}$ of film was analysed by Tauc plot from the absorbance spectra obtained using a Cary Varian 5000 UV-visible-NIR spectrometer. The thin-film electrical property and stoichiometry were characterised by a home-made four-point probe and EDX (Zeiss Evo), respectively. A home-built Hall measurement system using the van der Pauw configuration at room temperature was used to 
measure the carrier concentration and mobility of films. $S$ of Bi-Te films was measured along the in-plane direction at RT by an MMR Tech. Seebeck effect system at $300 \mathrm{~K}$ under nitrogen atmosphere applying temperature differences from 0.1 to $0.45 \mathrm{~K}$, determined by the ratio of an output voltage $(\Delta V)$ generated across the film surface to the temperature difference $(\Delta T)$. The $P F$ of Bi-Te films was evaluated from $S$ and $\rho$ using the relation in eq. (1).

The sample-to-sample variation was already considered in these characterisations. The run-torun variation was analysed for thickness (4.4\%), electrical resistivity (3\%) and Seebeck (1.5\%) in four independent batches.

\section{Results and discussion}

\subsection{Surface topography}

During initial deposition, grains of the deposited material can form isolated islands on the substrate, depending on the wetting of the substrate [58], and the sputtering power and species mobility [59]. Under deposition onto a heated substrate, $\mathrm{Bi}_{2} \mathrm{Te}_{3}$ films grow by a $2 \mathrm{D}$ layer-bylayer growth mode [36-38] or a 2D step flow growth mode [39,40]. The surface roughness should remain constantly that of the substrate for step-flow growth mode. In layer-by-layer growth the roughness should oscillate between the highest, just before island coalescence in each layer, and the lowest, at the formation of the next integer layer [60]. In contrast, 3D island growth mode is often observed for metal deposition onto polymer substrates at RT e.g. [61,62]. In this study we consider the effect of RT deposition with short deposition times and thus characterised the layers to establish confidently the nature of the first stages of growth of the films in this case. Figure 1 shows the surface morphology for Bi-Te films grown by RT sputtering. AFM images reveal a process of the initial small-islands combining to form larger, subdivided, grains. The line profiles show how the islands of deposition become taller and merge as more material is deposited. The root mean square roughness (RMSR), as shown in Figure 2, provides a simple quantification of this effect. The thinnest films are deposited 
through oblique deposition: the 1-min sample shows a significantly higher RMSR than the uncoated substrate because the film is partially coated with islands of coated material protruding a couple of nm above the uncoated substrate surface leading to an increase in roughness over that of the substrate, while after 3-min coating the islands are impinging, giving rise to an overall slightly smoother surface which then roughens again as the film thickens further after 5 minutes deposition. These cannot be compared directly to the samples mounted directly opposite the target as the angle of deposition may influence the morphology and properties, but from the samples mounted directly opposite the target, it is clear that as the film thickness grows further, the surface roughness then increases, as previously reported for island-growth mode [63].

For the shortest deposition times, therefore, the layers may not form a complete coating with the islands fully impinged. To further examine this, both SEM and AFM phase images are obtained for 1-min coating (Figure 3), as contrasted with a much thicker layer (5-min deposition sample) that is expected to form a complete layer. SEM images clearly demonstrate the morphological difference of films under different thickness. Unlike the big grains observed in the thick film, the 1-min coating shows a small-island structural morphology. The AFM phase image strongly suggests an island-growth period for 1-min coating due to the strong contrast between the coating and the relatively compliant substrate. The acrylate layers will tend to smooth over the larger-scale defects on the polymer substrate to smooth the film (Figure 2a). The relationship between RMSR and the film thickness is summarised in the schematic in Figure $2 \mathrm{~b}$. At a very high film thickness the film surface roughness is assumed to tend towards some equilibrium value (dashed line). 

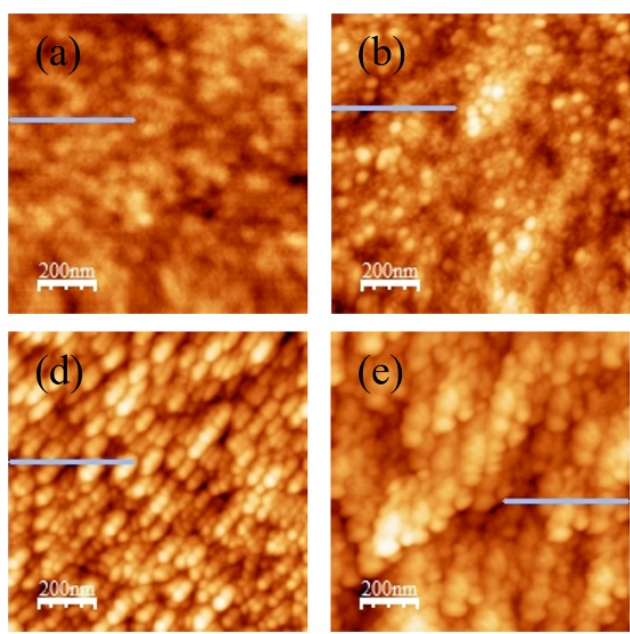
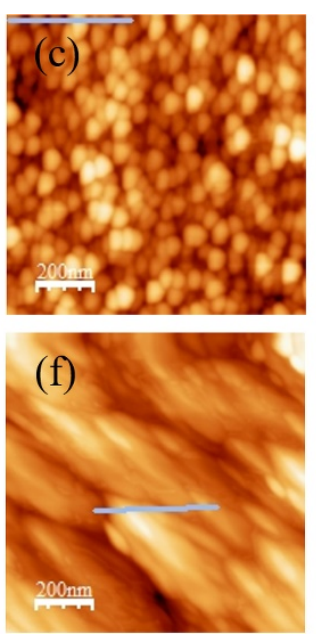

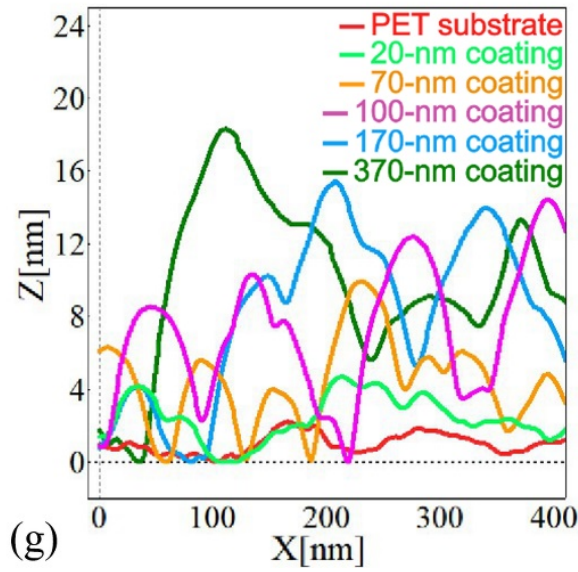

(g)

Figure 1. (a-f) AFM topography images and ( $g$ ) example line profiles (from positions indicated by grey lines in the images) with the image size of $1 \mu m \times 1 \mu \mathrm{m}$ for Bi-Te films deposited directly opposite the target: (a) the polymer substrate, (b) 1-min coating, (c) 2-min coating, (d) 3-min coating, (e) 5-min coating, (f) 10-min coating.
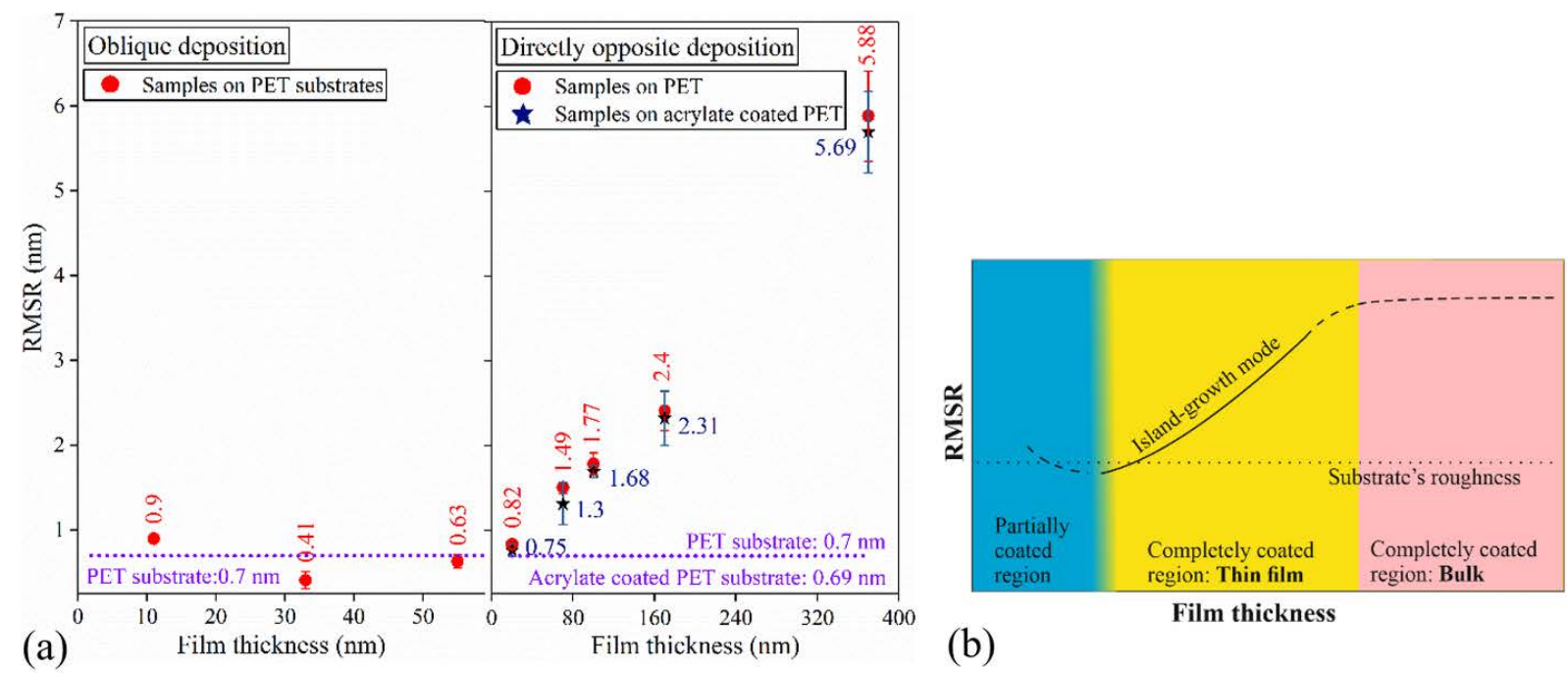

(b)

Figure 2. (a) RMSR of Bi-Te films (The error bars represent a standard deviation, which are obtained from at least eight $1 \mu \mathrm{m} \times 1 \mu \mathrm{m}$ images); (b) Schematic of the variation of RMSR with thickness of films grown by RT sputtering (The solid line is analysed from the experimental results, while the dashed line is estimated. The dotted line indicates the roughness of the substrate). 


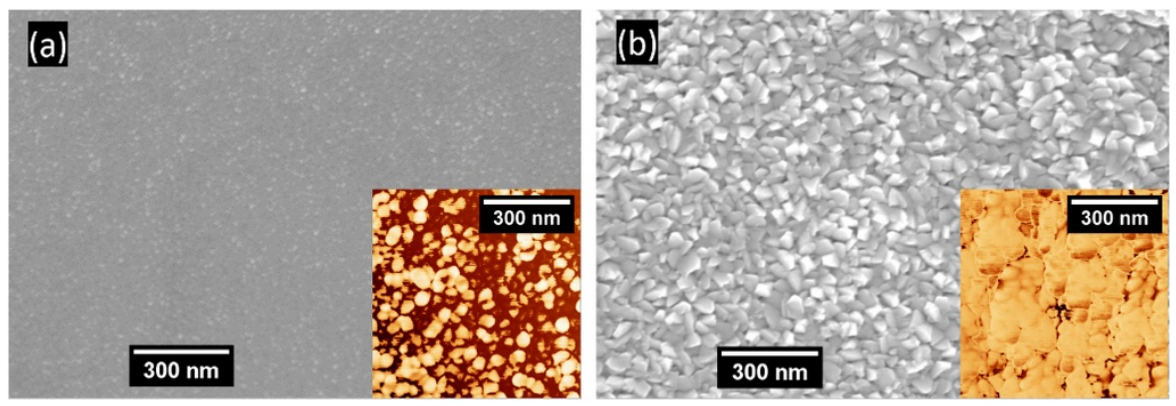

Figure 3. SEM images and AFM phase images (inset) of (a) 1-min and (b) 5-min films deposited directly opposite the target.

\subsection{Elemental Composition}

When considering the properties of a layer as a function of thickness it is important to consider any possible change in stoichiometry. Figure 4 shows a change in elemental ratio, as measured by EDX, of Te and Bi with deposition time (i.e. the film thickness). All the asdeposited films were found to be Te-rich compared to the sputtering target as reported elsewhere [64], in this case for RF sputtering, where for high powers Te content could reach as high as $90 \%$. An off-stoichiometric Bi-Te film does not directly produce poor TE properties because an excess Te content acts as a major source of electron donors [19].

The Te:Bi ratio changes with thickness (Figure 4) perhaps due to a different sticking coefficient to the substrate, giving a rise in Te near the interface which is averaged over the different thicknesses as measured by the penetration depth of the EDX. In addition, time spent passing under the sputter target (i.e. deposition time) may result in preferential removal of one element from the coating [65]. Such etching process could be either from the plasma region or from the high energy backscattered Ar neutrals from the sputter target. The Te atom in the film is preferred to be removed due to its poorer binding energy, smaller atomic size and atomic mass, hence, the Te content in the film decreases as the film grows.

The effect of stoichiometry on electrical properties is worth considering for our Bi-Te films. Goncalves et al. [66] carried out a stoichiometric study of $\mathrm{Bi}_{2} \mathrm{Te}_{3}$ films and found that, at lowtemperature deposition (in this case by evaporation), as the Te content of the film increases in 
the approximate range of Te:Bi ratio 1.5-2.5, the magnitude of $S$ decreases and $\rho$ increases leading to a decrease in $P F$. However, our results are the reverse of their observation. Goncalves et al. used a constant film thickness, so the reversed trend with stoichiometry suggests that the origin of the change in electrical behaviour observed here is the variation in film thickness and/or morphology, not the stoichiometry. Although the difference in deposition method may preclude a direct comparison with Goncalves’ films, our measurements of carrier concentration (Figure 7a) also suggest that stoichiometry is not a dominant effect: we would expect to see a significant increase in carrier concentration with Te content [19], but in our case the thinnest sample has both the greatest Te content and lowest carrier concentration.

Due to sputtering under a high vacuum condition (rather than an ultra-high vacuum), oxygen impurity in the film is of concern, e.g. acting as a dopant [67]. Since the interaction volume of SEM is even larger than the film thickness, measuring oxygen content by EDX in our film means that oxygen signals from the polymer substrate cannot be avoided. Hence, we made the Bi-Te films on Si wafer. In EDX analysis, there is no oxygen signal detected from samples on Si. However, it is noted that oxygen reading could be within the noise background and detection of EDX for a light element such as oxygen is poor. In contrast to an (54 - 7 at.\% from thin to thick films) oxygen detected in samples on the polymer substrate, there is no oxygen detected in samples on Si indicating that the presence of oxygen in our Bi-Te film is unlikely. In addition, since the TE performance of our films is consistent with $[31,68]$, it is not an overriding influence the film even were oxygen present, and the trends with thickness we report would not be explained by the oxygen content. 


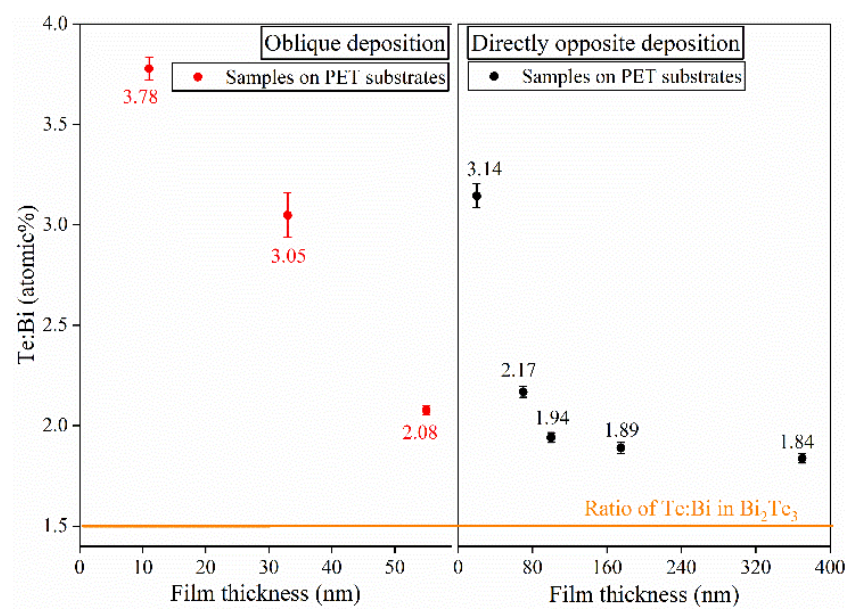

Figure 4. Elemental ratio of Te and Bi as a function of deposition time (The error bars are standard error from at least six independent points in six different locations on a large-area sample).

\subsection{Thermoelectric parameters}

\subsubsection{Resistivity}

The variation of $\rho, S$ and $P F$ as a function of film thickness has been measured (Figure 5). Both Seebeck and Hall measurements confirm all the films to be n-type. Both deposition conditions show a sharp increase in $\rho$ for the very thinnest films, commensurate with islands of material not fully connected to one another. Under this condition any conductivity is due to a small number of paths of connectivity between the islands, with poorly conducting, disordered or extremely thin/no material between. Dheepa et al. [69] concluded that the resistivity of $\mathrm{Bi}$-Te films deposited at $\mathrm{RT}$, as a result of island-growth, depended upon the tunneling of charge carriers between the islands once they were separated by only a few angstroms, but for the thinnest layers, where there are relatively few such connections, the resistivity would sharply increase in layers that are not made up of fully impinged islands. A sharp increase in resistance for very thin films observed in PbTe thermoelectric layers [70] was similarly linked to percolation and linking of islands of material as the amount of deposited material increased. 

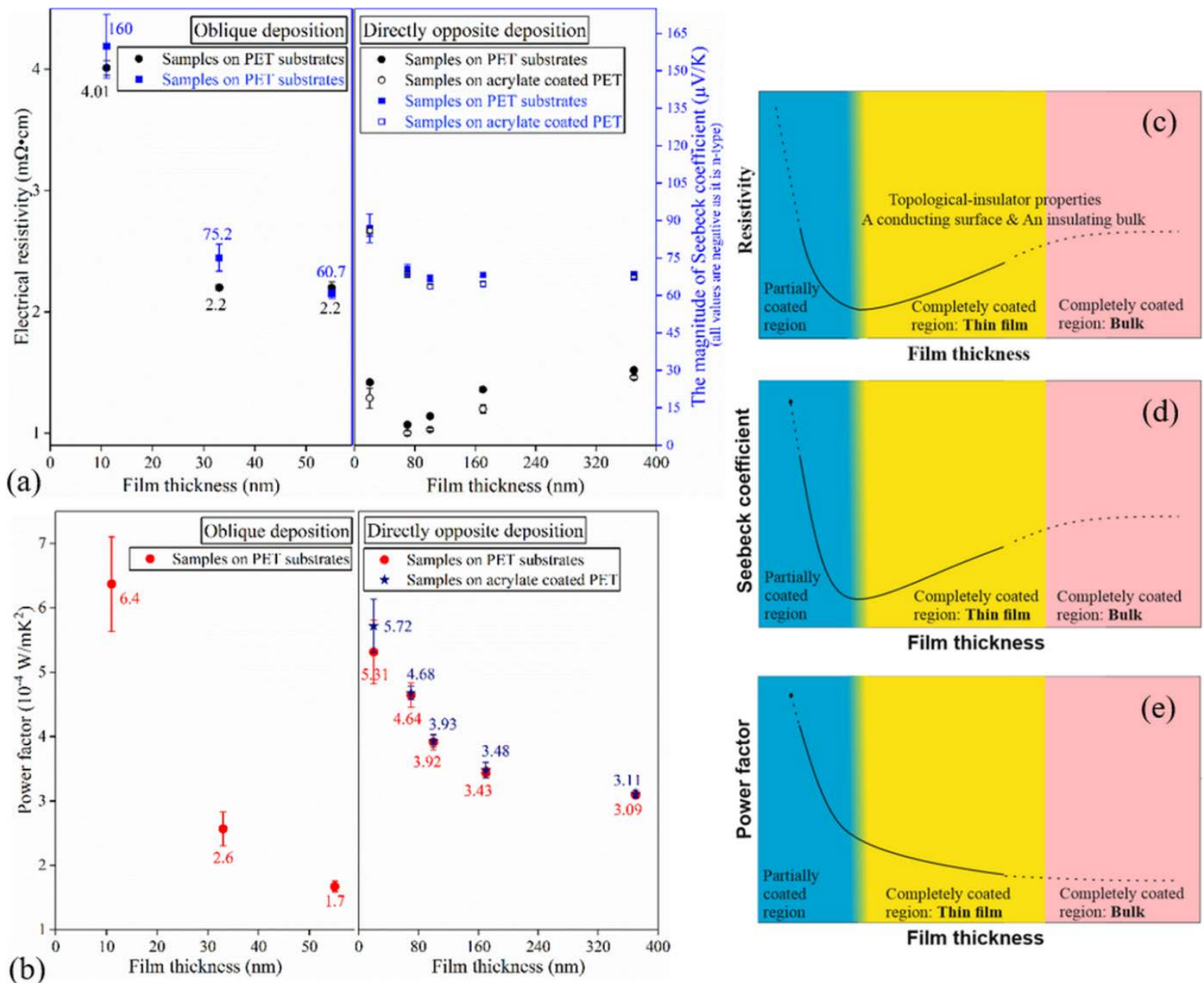

Figure 5. Plots of $\rho, S(a)$ and PF (b) for Bi-Te films with various deposition time, and schematic representations of the relationship between film thickness and $\rho(c), S(d)$, and PF (e) (The solid lines are analysed from the experimental result, while the dashed lines are estimated. The error bars are the standard deviation of at least six and three measurements at different locations for $S$ and $\rho$, respectively).

After a complete layer is formed, $\rho$ gradually increases in an approximately linear fashion, as represented in Figure 5c, as would be expected for a topological insulator until such thickness as the overall resistivity reflects that of the bulk. Changes in conductivity might also be influenced by changes in the contact with the four-point probe as the topography and morphology change, or changes in carrier mobility as a function of grain size [71] and degree of crystallinity [69,72]. 


\subsubsection{Seebeck coefficient}

The relationship between $S$ and film thickness can be described as illustrated in Figure $5 \mathrm{~d}$. $S$ is observed to increase sharply for the thinnest layers as was also observed for PdTe [70]. In this region the film thickness is small compared to the Bohr radius of $\mathrm{Bi}_{2} \mathrm{Te}_{3}$ (57 $\mathrm{nm}$ [73]). Indeed the $E_{g}$, as determined by Tauc plots from UV-vis data (Figure 6), is seen to increase significantly for the thinnest films, as would be expected from the effect of quantum confinement. A greater $E_{g}$ would be associated with a lower density of charge carriers (from intrinsic carrier generation) for a given temperature, which is expected to increase $S$. Quantum confinement in one dimension (for a thin film) would also lead to a change in density of states near the Fermi level which would change TE performance, and Dmitriev et al. 2010, [74] ascribed the higher $P F$ for thinner nanowires (confinement in two dimensions) to sharp features in the density of states resulting from the effect of quantum confinement. However, the trends in $S$ and $E_{g}$ we observed do not correlate well (i.e. the increase in $S$ is only observed for deposition time less than 3 mins), so this is not shown to be the primary explanation. We should also consider the effect of barrier scattering which is established as a reason for larger $S$ in thin films than bulk samples [75,76]. This scattering arises from potential barriers in the material, such as at grain boundaries, which stop the carriers of lower energy, increasing $S$.
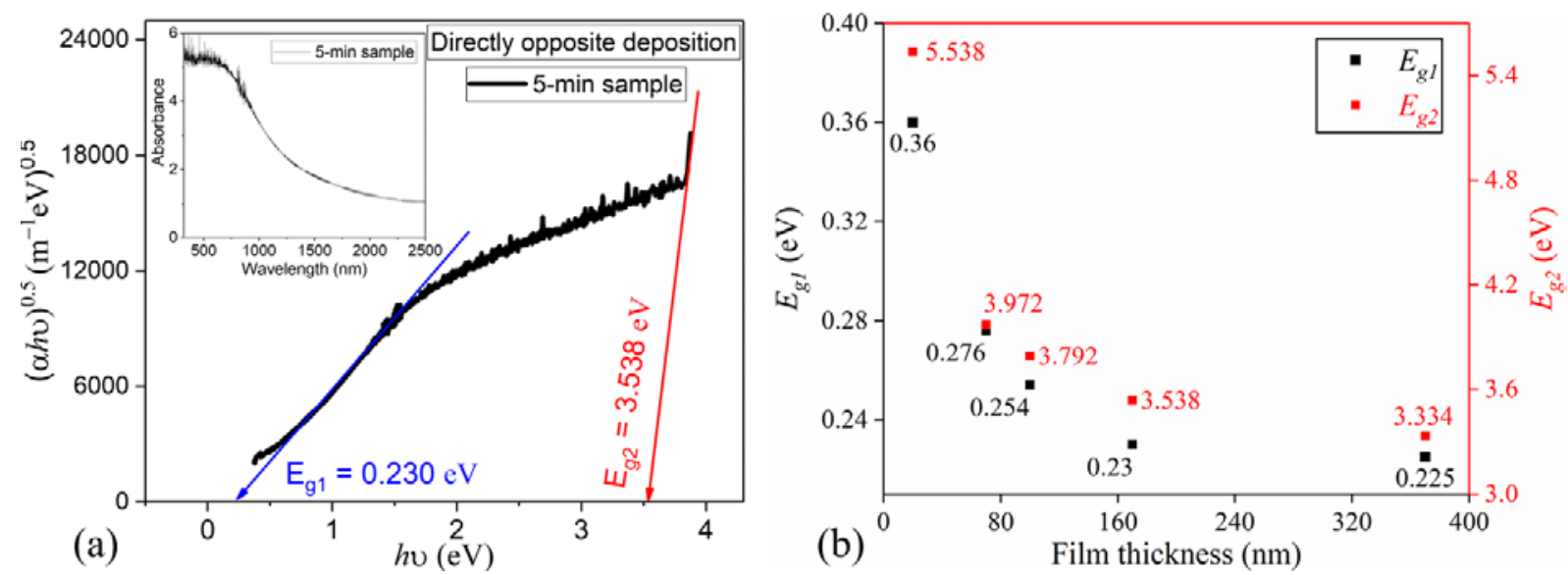

Figure 6. (a) Tauc plot of Bi-Te films under 5-min opposite deposition with (inset) corresponding absorbance spectra (where $\alpha$ is absorbance coefficient, hv is photon energy, 
$E_{g}$ is band gap) The primary $E_{g}$ is observed at around $0.23 \mathrm{eV}$, similar to other reports [35,77 ] and a second $E_{g}$ is seen corresponding to a gap of $3.54 \mathrm{eV}$ that has been reported by [78]; (b) The variation of two types of $E_{g}$ as a function of deposition time/film thickness.

The thinner films will have fewer high-quality connections between grains (good conducting material), with potentially poorly ordered material between (which would serve as a potential barrier). In this study, the XRD measurement was not obtained because the film is too thin to obtain sufficient signal, however the room-temperature sputtered Bi-Te film is not expected to be highly crystalline which has already been observed for thicker Bi-Te films in our previous study [72]. In the thinnest, partially coated, films, the material relies on a relatively low number of percolation pathways for conduction, and the intimate, ideal, electronic connection between neighbouring grains will not be made in many cases, thus a relatively large proportion of the moving charge will need to pass through higher potential barriers. To describe it in another way, the thinnest layers might be considered as an incomplete layer of a nanocomposite material of Bi-Te with inclusions of poor quality/no/high Te/oxidized-phase material between that would serve to give an energy filtering effect, stopping the low energy carriers, as above, as reported for nanocomposites [79,80]. Thus, $S$ increases as the carrier concentration decreases (the inverse relationship between $S$ and the carrier concentration has been reported by many studies $[9,74,81])$.

As a continuous layer is formed for thicker films, the carrier pathways become multitudinous (continuous) and so the sample no longer depends on such, imperfect, connections, as good connectivity between the better-quality material will be much more common with a reduction or removal of the carrier filtering effect.

\subsubsection{Carrier concentration and mobility}

The measured carrier concentration and carrier mobility taken from Hall measurements, Figure 7, do confirm a very substantial decrease in carrier concentration for the thinnest layer, 
due to the carrier filtering effect. The trends for thicker films, observed in both carrier concentration and mobility do give rise to an increase in resistivity with deposition time, as observed in Figure 5, and are expected to be influenced by stoichiometry (doping: increased Te doping in the thinner films is a source of electron donors [19]; oxygen doping could increase TE performance [67]), and grain size and crystallinity (larger grains $[4,6,78]$ and better crystallinity [72] have been observed in thicker layers). The observed decrease in carrier concentration (Figure 7a) with increased deposition time for the thicker films is consistent with the observed increase in $S$ (Figure 5a).
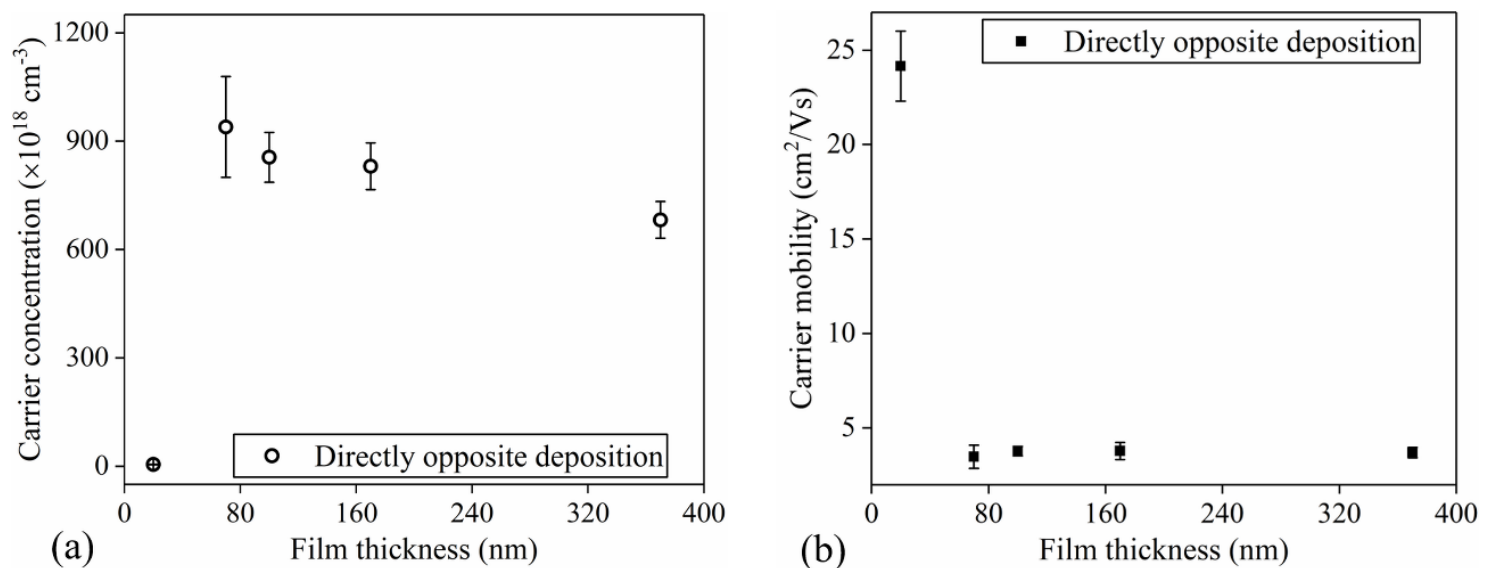

Figure 7. Variation of (a) carrier concentration and (b) carrier mobility of Bi-Te films as a function of sputtering time i.e. film thickness (The error bars represent a standard error, which are obtained from three measurements).

\subsection{Thermoelectric behaviour: Power factor}

The samples deposited directly opposite the target show much better $P F$ (product of $S^{2}$ and $\sigma$, as from eq. 1) than those deposited obliquely, even when in the same range of thickness, because of their lower $\rho$ but higher $S$. This can be attributable to a difference in the morphology (e.g. RMSR), uniformity and potentially stoichiometry of films deposited at different positions on the drum. Those films deposited on acrylate-coated PET have better PF than those deposited directly onto PET: the acrylate layer helps to smooth over scratches and 
other micro-scale defects on the PET surface that are likely to break the carrier's percolation path, as is reflected in their lower resistivity.

We have observed that very thin Bi-Te films exhibit better PF behaviour (Figure $5 b$ ). This is significant and encouraging, given that very thin layers are favoured for a high-throughput manufacture. This is the first such study for very thin Bi-Te films grown at RT in moving web deposition. From the experimental result, the relationship between $P F$ and the film thickness is proposed as shown in Figure 5e. The competition between the change in $\rho$ and the change in $S$ is the key. In the completely coated region, $P F$ increases as the film becomes thinner because the decrease in $\rho$ is larger, hence the change in $\rho$ dominates the change in $P F$. In the partially coated region, $P F$ increases as the film becomes thinner because the change in $S$ dominates the change in PF. Hence the coupling between $\rho$ and $S$ is different for the thinnest layers due to the different mechanisms involved, giving rise the relatively high $P F$ observed for the thinnest layer.

\section{Conclusion}

An experimental study has been carried out to investigate the TE behaviour of RT-sputtered Bi-Te thin films on polymer substrates in the thickness range of few nm - $370 \mathrm{~nm}$. AFM analysis revealed an island-growth mode of Bi-Te films deposited at RT by DC magnetron sputtering. Electrical resistivity was found to be the lowest as a complete coating is formed, and an almost linear relationship between electrical resistivity and the film thickness was observed thereafter, as expected for topological-insulator films. In addition to a possible lowdimensional quantum confinement effect, an energy barrier mechanism was proposed to explain the extremely high Seebeck coefficient at the partially coated region. The islandgrowth behaviour observed in these RT-deposited layers facilitated forming coatings which showed this energy barrier effect, as the layer-by-layer growth reported for high substrate 
temperature deposition would tend to give rise to good connectivity across the layer. The power factor was shown to increase substantially in very thin films, which has been explained by a quasi-decoupling of $S$ and $\rho$. Therefore, both a high deposition throughput and a high $P F$ could be fulfilled by using very thin TE materials, giving a boost to the commercialization of flexible TEGs even through the film sputtered at room temperature under high vacuum level is not highly crystallised and possibly involves oxidation phase for sputtering at such vacuum level (not an ultra-high vacuum level). The film surface features were improved by applying an acrylate smoothing layer to the substrate which could enhance power factor by improving the electrical resistivity of films.

\section{Acknowledgments}

This study was supported by the Engineering and Physical Sciences Research Council [grant number EP/M015173/1] via the Wearable and flexible technologies enabled by advanced thin-film manufacture and metrology (WAFT) Collaboration. The authors would like to acknowledge Oxford Materials Characterisation Services (OMSC) and David Cockayne Centre for Electron Microscopy for equipment access, especially thank to Dr Colin Johnston, Mrs Nicola Flanagan, Mrs Jennifer Holter and Mr Richard Turner for technical assistance.

\section{Reference}

[1] A. R. M. Siddique, S. Mahmud, and B. V. Heyst, "A review of the state of the science on wearable thermoelectric power generators (TEGs) and their existing challenges," Renewable and Sustainable Energy Reviews, vol. 73, pp. 730-744, 2017. https://doi.org/10.1016/j.rser.2017.01.177.

[2] D. Enescu, "Thermoelectric Energy Harvesting: Basic Principles and Applications," Green Energy Advances, 2019. https://doi.org/10.5772/intechopen.83495.

[3] M. H. Liao, K. C. Huang, F. A. Tsai, C. Y. Liu, C. Lien, and M. H. Lee, "Thickness dependence of electrical conductivity and thermo-electric power of 
Bi2.0Te2.7Se0.3/Bi0.4Te3.0Sb1.6 thermo-electric devices," AIP Advances, vol. 8, no. 1, pp. 015020-4, 2018. http://dx.doi.org/10.1063/1.5017252.

[4] J. Dheepa, R. Sathyamoorthy, and A. Subbarayan, "Optical properties of thermally evaporated Bi2Te3 thin films," Journal of Crystal Growth, vol. 274, no. 1, pp. 100-105, 2005. https://doi.org/10.1016/j.jcrysgro.2004.09.070.

[5] H. E. A. El-Sayed, "Structural and optical properties of thermally evaporated Bi2Te3 films," Applied Surface Science, vol. 250, no. 1-4, pp. 70-78, 2005. https://doi.org/10.1016/j.apsusc.2004.12.025.

[6] G. D. Deshmukh, S. M. Patil, S. S. Patil, and P. H. Pawar, "Effect of Film Thickness on Structural and Optical Properties of Bi2Te3 Thin Films," Journal of Chemical, Biological and Physical Sciences, vol. 5, no. 3, pp. 2769-2779, 2015. https://www.jcbsc.org/admin/get_filephy.php?id=335.

[7] L. Yucheng, A. J. Minnich, C. Gang, and R. Zhifeng, "Enhancement of Thermoelectric Figure of Merit by a Bulk Nanostructuring Approach," Advanced Functional Materials, vol. 20, no. 3, pp. 357-376, 2010. https://doi.org/10.1002/adfm.200901512.

[8] H. J. Goldsmid, "Thermoelectric refrigeration," Springer, 1964. http://www.springer.com/gb/book/9781489957252.

[9] C Wood, "Materials for thermoelectric energy conversion," Reports on Progress in Physics, vol. 51, no. 4, pp. 459-539, 1988. https://doi.org/10.1088/0034-4885/51/4/001.

[10] Mi-Kyung Han, Yingshi Jin, Da-Hee Lee, and Sung-Jin Kim, "Thermoelectric Properties of Bi2Te3: CuI and the Effect of Its Doping with Pb Atoms," Materials, vol. 10, no. 11, pp. 1235, 2017. https://doi.org/10.3390/ma10111235.

[11] L. Zhao, B. Zhang, J. Li, M. Zhou, W. Liu, and J. Liu, "Thermoelectric and mechanical properties of nano-SiC-dispersed $\mathrm{Bi} 2 \mathrm{Te} 3$ fabricated by mechanical alloying and spark plasma sintering," Journal of Alloys and Compounds, vol. 455, no. 1-2, pp. 259-264, 2008. https://doi.org/10.1016/j.jallcom.2007.01.015.

[12] H. Mun, S. Choi, K. H. Lee, and S. W. Kim, "Boundary Engineering for the Thermoelectric Performance of Bulk Alloys Based on Bismuth Telluride," ChemSusChem, vol. 8, no. 14, pp. 2312-2326, 2015. http://dx.doi.org/10.1002/cssc.201403485.

[13] Y. Liu, M. Zhou, and J. He, "Towards higher thermoelectric performance of Bi2Te3 via defect engineering," Scripta Materialia, vol. 111, pp. 39-43, 2016. http://dx.doi.org/10.1016/j.scriptamat.2015.06.031.

[14] A. J. Minnich, M. S. Dresselhaus, Z. F. Ren, and G. Chen, "Bulk nanostructured thermoelectric materials: current research and future prospects," Energy \& Environmental Science, vol. 2, no. 5, pp. 466-479, 2009. http://dx.doi.org/10.1039/b822664b.

[15] Y. Du, J. Xu, B. Paul, and P. Eklund, "Flexible thermoelectric materials and devices," Applied Materials Today, vol. 12, pp. 2018. https://doi.org/10.1016/j.apmt.2018.07.004. 
[16] A. Dauscher, A. Thomy, and H. Scherrer, "Pulsed laser deposition of Bi2Te3 thin films," Thin Solid Films, vol. 280, no. 1-2, pp. 61-66, 1996. https://doi.org/10.1016/00406090(95)08221-2.

[17] A. Bailini, F. Donati, M. Zamboni, V. Russo, M. Passoni, C. S. Casari, A. Li Bassi, and C. E. Bottani, "Pulsed laser deposition of Bi2Te3 thermoelectric films," Applied Surface Science, vol. 254, no. 4, pp. 1249-1254, 2007. https://doi.org/10.1016/j.apsusc.2007.09.039.

[18] A. Boyer and E. Cissé, "Properties of thin film thermoelectric materials: application to sensors using the Seebeck effect," Materials Science \& Engineering B, vol. 13, no. 2, pp. 103111, 1992. https://doi.org/10.1016/0921-5107(92)90149-4.

[19] S. Cho, Y. Kim, A. DiVenere, G. K. Wong, J. B. Ketterson, and J. R. Meyer, "Antisite defects of Bi2Te3 thin films," Applied Physics Letters, vol. 75, no. 10, pp. 1401-1403, 1999. https://doi.org/10.1063/1.124707.

[20] A. Giani, F. Pascal-Delannoy, A. Boyer, A. Foucaran, M. Gschwind, and P. Ancey, "Elaboration of Bi2Te3 by metal organic chemical vapor deposition," Thin Solid Films, vol. 303, no. 1, pp. 1-3, 1997. https://doi.org/10.1016/S0040-6090(97)00089-8.

[21] A. Boulouz, M. Boulouz, A. Giani, F. Pascal-Delannoy, A. Foucaran, and A. Boyer, "Preparation and characterization of MOCVD bismuth telluride thin films," Journal of Crystal Growth, vol. 194, no. 3, pp. 336-341, 1998. https://doi.org/10.1016/S0022-0248(98)00690-3.

[22] Y. Miyazaki and T. Kajitani, "Preparation of Bi2Te3 films by electrodeposition," Journal of Crystal Growth, vol. 229, no. 1-4, pp. 542-546, 2001. https://doi.org/10.1016/S00220248(01)01225-8.

[23] J. Dheepa, R. Sathyamoorthy, and A. Subbarayan, "Optical properties of thermally evaporated Bi2Te3 thin films," Journal of Crystal Growth, vol. 274, no. 1, pp. 100-105, 2005. https://doi.org/10.1016/j.jcrysgro.2004.09.070.

[24] R. O. -Ndong, H. G. anga, A. G. ani, and A. F. aran, "Anemometer based on peltier effect deposed by flash evaporation," International Journal of Advanced Research in Electrical, Electronics and Instrumentation Engineering, vol. 4, no. 1, pp. 42-49, 2015. https://doi.org/10.15662/ijareeie.2015.0401040.

[25] F. Vlklein, V. Baier, U. Dillner, and E. Kessler, "Transport properties of flash-evaporated (Bi1-xSbx)2Te3 films I: Optimization of film properties," Thin Solid Films, vol. 187, no. 2, pp. 253-262, 1990. https://doi.org/10.1016/0040-6090(90)90047-H.

[26] X. Duan and Y. Jiang, "Annealing effects on the structural and electrical transport properties of n-type Bi2Te2.7Se0.3 thin films deposited by flash evaporation," Applied Surface Science, vol. 256, no. 24, pp. 7365-7370, 2010. https://doi.org/10.1016/j.apsusc.2010.05.069.

[27] H. Zou, D. M. Rowe, and G. Min, "Preparation and characterization of p-type Sb2Te3 and n-type Bi2Te3 thin films grown by coevaporation," Journal of Vacuum Science \& Technology A: Vacuum, Surfaces, and Films, vol. 19, no. 3, pp. 899-903, 2001. https://doi.org/10.1116/1.1354600. 
[28] H. Zou, D. M. Rowe, and G. Min, "Growth of p- and n-type bismuth telluride thin films by co-evaporation," Journal of Crystal Growth, vol. 222, no. 1, pp. 82-87, 2001. https://doi.org/10.1016/S0022-0248(00)00922-2.

[29] H. Noro, K. Sato, and H. Kagechika, "The thermoelectric properties and crystallography of Bi-Sb-Te-Se thin films grown by ion beam sputtering," Journal of Applied Physics, vol. 73, no. 3, pp. 1252-1260, 1993. https://doi.org/10.1063/1.353266.

[30] D. Kim, E. Byon, G. Lee, and S. Cho, "Effect of deposition temperature on the structural and thermoelectric properties of bismuth telluride thin films grown by co-sputtering," Thin Solid Films, vol. 510, no. 1, pp. 148-153, 2006. https://doi.org/10.1016/j.tsf.2005.12.306.

[31] H. Huang, W. Luan, and S. Tu, "Influence of annealing on thermoelectric properties of bismuth telluride films grown via radio frequency magnetron sputtering," Thin Solid Films, vol. 517, no. 13, pp. 3731-3734, 2009. https://doi.org/10.1016/j.tsf.2009.01.015.

[32] Y. Zhou, L. Li, Q. Tan, and J. Li, "Thermoelectric properties of Pb-doped bismuth telluride thin films deposited by magnetron sputtering," Journal of Alloys and Compounds, vol. 590, pp. 362-367, 2014. https://doi.org/10.1016/j.jallcom.2013.12.136.

[33] M. J. Madou, "Manufacturing techniques for microfabrication and nanotechnology," , pp. 403-404, 2011. https://doi.org/10.1201/9781439895306.

[34] Taylor, Cliffordl, Bjornard, Erikj, Valiska, and Michaelj, "Film thickness uniformity control apparatus for in-line sputtering systems," US patents, 1992. https://patents.google.com/patent/US5156727.

[35] J. Bahk, H. Fang, K. Yazawa, and A. Shakouri, "Flexible thermoelectric materials and device optimization for wearable energy harvesting," J. Mater. Chem. C, vol. 3, no. 4, pp. 13621374, 2015. https://doi.org/10.1039/c5tc01644d.

[36] H. W. Liu, H. T. Yuan, N. Fukui, L. Zhang, J. F. Jia, Y. Iwasa, M. W. Chen, T. Hashizume, T. Sakurai, and Q. K. Xue, "Growth of topological insulator Bi2Te3 ultrathin films on Si (111) investigated by low-energy electron microscopy," Crystal Growth \& Design, vol. 10, no. 10, pp. 4491-4493, 2010. https://doi.org/10.1021/cg1007457.

[37] Y. Y. Li, W. Guang, X. G. Zhu, M. H. Liu, Y. Cun, C. Xi, Y. Y. Wang, H. Ke, L. L. Wang, X. C. Ma, H. J. Zhang, D. Xi, F. Zhong, X. C. Xie, L. Ying, X. L. Qi, J. F. Jia, S. C. Zhang, and Q. K. Xue, "Intrinsic Topological Insulator Bi2Te3 Thin Films on Si and Their Thickness Limit," Advanced Materials, vol. 22, no. 36, pp. 4002-4007, 2010. https://doi.org/10.1002/adma.201000368.

[38] J. Krumrain, G. Mussler, S. Borisova, T. Stoica, L. Plucinski, C. M. Schneider, and D. Grtzmacher, "MBE growth optimization of topological insulator Bi2Te3 films," Journal of Crystal Growth, vol. 324, no. 1, pp. 115-118, 2011. https://doi.org/10.1016/j.jcrysgro.2011.03.008.

[39] S. Borisova, J. Krumrain, M. Luysberg, G. Mussler, and D. Gruľtzmacher, "Mode of growth of ultrathin topological insulator Bi2Te3 films on Si (111) substrates," Crystal Growth \& Design, vol. 12, no. 12, pp. 6098-6103, 2012. https://doi.org/10.1021/cg301236s. 
[40] P. Schönherr, T. Tilbury, H. Wang, A. A. Haghighirad, V. Srot, P. A. van Aken, and T. Hesjedal, "Step-flow growth of Bi2Te3 nanobelts," Crystal Growth \& Design, vol. 16, no. 12, pp. 6961-6966, 2016. https://doi.org/10.1021/acs.cgd.6b01147.

[41] J. Shen, T. Zhu, X. Zhao, S. Zhang, S. Yang, and Z. Yin, "Recrystallization induced in situ nanostructures in bulk bismuth antimony tellurides: a simple top down route and improved thermoelectric properties," Energy \& Environmental Science, vol. 3, no. 10, pp. 1519-1523, 2010. https://doi.org/10.1039/C0EE00012D.

[42] C. V. Manzano, B. Abad, M. M. Rojo, Y. R. Koh, S. L. Hodson, A. M. L. Martinez, X. $\mathrm{Xu}, \mathrm{A}$. Shakouri, T. D. Sands, and T. Borca-Tasciuc, "Anisotropic effects on the thermoelectric properties of highly oriented electrodeposited Bi2Te3 films," Scientific reports, vol. 6, pp. 19129, 2016. https://doi.org/10.1038/srep19129.

[43] E. E. Antonova and D. C. Looman, "Finite elements for thermoelectric device analysis in ANSYS," , pp. 215-218, 2005. https://doi.org/10.1109/ICT.2005.1519922.

[44] J. P. Fleurial, L. Gailliard, R. Triboulet, H. Scherrer, and S. Scherrer, "Thermal properties of high quality single crystals of bismuth telluride-Part I: Experimental characterization," Journal of Physics and Chemistry of Solids, vol. 49, no. 10, pp. 1237-1247, 1988. https://doi.org/10.1016/0022-3697(88)90182-5.

[45] J. A. Thornton, "Influence of apparatus geometry and deposition conditions on the structure and topography of thick sputtered coatings," Journal of Vacuum Science and Technology, vol. 11, no. 4, pp. 666-670, 1974. https://doi.org/10.1116/1.1312732.

[46] G. Wang, X. Zhu, Y. Sun, Y. Li, T. Zhang, J. Wen, X. Chen, K. He, L. Wang, X. Ma, J. Jia, S. B. Zhang, and Q. Xue, "Topological insulator thin films of Bi2Te3 with controlled electronic structure," Advanced materials (Deerfield Beach, Fla.), vol. 23, no. 26, pp. 29292932, 2011. https://doi.org/10.1002/adma.201100678.

[47] H. Zhang, C. Liu, X. Qi, X. Dai, Z. Fang, and S. Zhang, "Topological insulators in Bi2Se3, Bi2Te3 and Sb2Te3 with a single Dirac cone on the surface," Nature Physics, vol. 5, no. 6, pp. 438-442, 2009. https://doi.org/10.1038/nphys1270.

[48] S. Barua and K. P. Rajeev, "Status of surface conduction in topological insulators," AIP Advances, vol. 4, no. 1, pp. 017135-6, 2014. http://dx.doi.org/10.1063/1.4864058.

[49] J. G. Analytis, J. Chu, Y. Chen, F. Corredor, R. D. McDonald, Z. X. Shen, and I. R. Fisher, "Bulk Fermi surface coexistence with Dirac surface state inBi2Se3: A comparison of photoemission and Shubnikov-de Haas measurements," Physical Review B, vol. 81, no. 20, 2010. https://doi.org/10.1103/PhysRevB.81.205407.

[50] A. A. Taskin, Z. Ren, S. Sasaki, K. Segawa, and Y. Ando, "Observation of dirac holes and electrons in a topological insulator," Physical review letters, vol. 107, no. 1, pp. 016801, 2011. https://doi.org/10.1103/PhysRevLett.107.016801.

[51] Y. S. Kim, M. Brahlek, N. Bansal, E. Edrey, G. A. Kapilevich, K. Iida, M. Tanimura, Y. Horibe, S. Cheong, and S. Oh, "Thickness-dependent bulk properties and weak antilocalization effect in topological insulator Bi2Se3," Physical Review B, vol. 84, no. 7, 2011. https://doi.org/10.1103/PhysRevB.84.073109. 
[52] N. Bansal, Y. S. Kim, M. Brahlek, E. Edrey, and S. Oh, "Thickness-independent transport channels in topological insulator Bi2Se3 thin films," PhysRevLett., 2011. https://doi.org/10.1103/PhysRevLett.109.116804.

[53] B. Xia, P. Ren, A. Sulaev, P. Liu, S. Shen, and L. Wang, "Indications of surface-dominated transport in single crystalline nanoflake devices of topological insulator Bi1.5Sb0.5Te1.8Se1.2," Physical Review B, vol. 87, no. 8, 2013. https://doi.org/10.1103/PhysRevB.87.085442.

[54] H. Osterhage, J. Gooth, B. Hamdou, P. Gwozdz, R. Zierold, and K. Nielsch, "Thermoelectric properties of topological insulator Bi2Te3, Sb2Te3, and Bi2Se3 thin film quantum wells," Applied Physics Letters, vol. 105, no. 12, pp. 123117, 2014. https://doi.org/10.1063/1.4896680.

[55] N. Mingo, "Thermoelectric figure of merit of II-VI semiconductor nanowires," Applied Physics Letters, vol. 85, no. 24, pp. 5986-5988, 2004. http://dx.doi.org/10.1063/1.1829391.

[56] E. I. Rogacheva, A. V. Budnik, O. N. Nashchekina, A. V. Meriuts, and M. S. Dresselhaus, "Quantum Size Effects in Transport Properties of Bi2Te3 Topological Insulator Thin Films," Journal of Electronic Materials, vol. 46, no. 7, pp. 3949-3957, 2017. http://dx.doi.org/10.1007/s11664-017-5561-2.

[57] Z. Ding, G. A. W. Abbas, H. E. Assender, J. J. Morrison, S. G. Yeates, E. R. Patchett, and D. M. Taylor, "Vacuum production of OTFTs by vapour jet deposition of dinaphtho[2,3-b:2',3'f]thieno[3,2-b]thiophene (DNTT) on a lauryl acrylate functionalised dielectric surface," Organic Electronics, vol. 31, pp. 90-97, 2016. https://doi.org/10.1016/j.orgel.2016.01.024.

[58] R. Lazzari and J. Jupille, "Silver layers on oxide surfaces: morphology and optical properties," Surface Science, vol. 482-485, pp. 823-828, 2001. https://doi.org/10.1016/S00396028(01)00935-9.

[59] J. H. Je, T. S. Kang, and D. Y. Noh, "Epitaxial and island growth of Ag/Si(001) by rf magnetron sputtering," Journal of Applied Physics, vol. 81, no. 10, pp. 6716-6722, 1997. https://doi.org/10.1063/1.365213.

[60] H. Brune, "Growth Modes," Encyclopedia of Materials: Science and Technology, Sect. 1.9, Physical Properties of Thin Films and Artificial Multilayers, pp. 3683-3693, 2001. https://infoscience.epfl.ch/record/135795/.

[61] F. Ruffino, V. Torrisi, G. Marletta, and M. Grimaldi, "Growth morphology of nanoscale sputter-deposited Au films on amorphous soft polymeric substrates," Applied Physics A, vol. 103, no. 4, pp. 939-949, 2011. https://doi.org/10.1007/s00339-011-6413-1.

[62] Matthias Schwartzkopf, Gonzalo Santoro, Calvin J Brett, André Rothkirch, Oleksandr Polonskyi, Alexander Hinz, Ezzeldin Metwalli, Yuan Yao, Thomas Strunskus, Franz Faupel, Peter Müller-Buschbaum, and Stephan V Roth, "Real-Time Monitoring of Morphology and Optical Properties during Sputter Deposition for Tailoring Metal-Polymer Interfaces," ACS applied materials \& interfaces, vol. 7, no. 24, pp. 13547-13556, 2015. https://doi.org/10.1021/acsami.5b02901. 
[63] I. Petrov, P. B. Barna, L. Hultman, and J. E. Greene, "Microstructural evolution during film growth," Journal of Vacuum Science \& Technology A: Vacuum, Surfaces, and Films, vol. 21, no. 5, pp. S117-S128, 2003. http://dx.doi.org/10.1116/1.1601610.

[64] M. Goto, M. Sasaki, Y. Xu, T. Zhan, Y. Isoda, and Y. Shinohara, "Control of p-type and n-type thermoelectric properties of bismuth telluride thin films by combinatorial sputter coating technology," Applied Surface Science, vol. 407, pp. 405-411, 2017. https://doi.org/10.1016/j.apsusc.2017.02.187.

[65] J. M. Gregoire, M. B. Lobovsky, M. F. Heinz, F. J. DiSalvo, and R. B. van Dover, "Resputtering phenomena and determination of composition in codeposited films," Physical Review B, vol. 76, no. 19, 2007. https://doi.org/10.1103/PhysRevB.76.195437.

[66] L. M. Goncalves, C. Couto, P. Alpuim, A. G. Rolo, F. Völklein, and J. H. Correia, "Optimization of thermoelectric properties on $\mathrm{Bi} 2 \mathrm{Te} 3$ thin films deposited by thermal coevaporation," Thin Solid Films, vol. 518, no. 10, pp. 2816-2821, 2010. http://dx.doi.org/10.1016/j.tsf.2009.08.038.

[67] T. Van Quang and M. Kim, "Enhancement of the thermoelectric performance of oxygen substituted bismuth telluride," Journal of Applied Physics, vol. 122, no. 24, pp. 245104, 2017. http://dx.doi.org/10.1063/1.5006233.

[68] K. Singkaselit, A. Sakulkalavek, and R. Sakdanuphab, "Effects of annealing temperature on the structural, mechanical and electrical properties of flexible bismuth telluride thin films prepared by high-pressure RF magnetron sputtering," Advances in Natural Sciences: Nanoscience and Nanotechnology, vol. 8, no. 3, pp. 35002, 2017. https://doi.org/10.1088/20436254/aa7222.

[69] J. Dheepa, R. Sathyamoorthy, S. Velumani, A. Subbarayan, K. Natarajan, and P. J. Sebastian, "Electrical resistivity of thermally evaporated bismuth telluride thin films," Solar Energy Materials and Solar Cells, vol. 81, no. 3, pp. 305-312, 2004. https://doi.org/10.1016/j.solmat.2003.11.008.

[70] E. I. Rogacheva, I. M. Krivulkin, O. N. Nashchekina, A. Y. Sipatov, V. A. Volobuev, and M. S. Dresselhaus, "Percolation transition of thermoelectric properties in PbTe thin films," Applied Physics Letters, vol. 78, no. 21, pp. 3238-3240, 2001. https://doi.org/10.1063/1.1357809.

[71] M. Bouderbala, S. Hamzaoui, B. Amrani, A. H. Reshak, M. Adnane, T. Sahraoui, and M. Zerdali, "Thickness dependence of structural, electrical and optical behaviour of undoped ZnO thin films," Physica B: Condensed Matter, vol. 403, no. 18, pp. 3326-3330, 2008. https://doi.org/10.1016/j.physb.2008.04.045.

[72] K. A. Morgan, T. Tang, I. Zeimpekis, A. Ravagli, C. Craig, J. Yao, Z. Feng, D. Yarmolich, C. Barker, H. Assender, and D. W. Hewak, "High-throughput physical vapour deposition flexible thermoelectric generators," Scientific Reports, vol. 9, no. 1, pp. 1-9, 2019. https://doi.org/10.1038/s41598-019-41000-y.

[73] R. P. Panmand, G. Kumar, S. M. Mahajan, N. Shroff, B. B. Kale, and S. W. Gosavi, "Growth of Bi2Te3 quantum dots/rods in glass: a unique highly stable nanosystem with novel functionality for high performance magneto optical devices," Physical chemistry chemical physics : PCCP, vol. 14, no. 47, pp. 16236, 2012. https://doi.org/10.1039/c2cp43169f. 
[74] A. V. Dmitriev and I. P. Zvyagin, "Current trends in the physics of thermoelectric materials," Physics-Uspekhi, vol. 53, no. 8, pp. 789-803, 2010. https://doi.org/10.3367/UFNe.0180.201008b.0821.

[75] S. B. A. Atakulov and A. N. Shamsiddinov, "The problem of transport phenomena in polycrystalline semiconductor thin films with potential barriers in the case where the carriers gas degenerated," Solid State Communications, vol. 56, no. 2, pp. 215-219, 1985. https://doi.org/10.1016/0038-1098(85)90744-6.

[76] K. Kishimoto, M. Tsukamoto, and T. Koyanagi, "Temperature dependence of the Seebeck coefficient and the potential barrier scattering of n-type PbTe films prepared on heated glass substrates by rf sputtering," Journal of Applied Physics, vol. 92, no. 9, pp. 5331, 2002. https://doi.org/10.1063/1.1512964.

[77] M. Shaik and I. A. Motaleb, "Investigation of the optical properties of PLD-grown Bi2Te3 and Sb2Te3," IEEE International Conference on Electro-Information Technology, EIT 2013, pp. 1-6, 2013. https://doi.org/10.1109/EIT.2013.6632708.

[78] S. M. Elahi, A. Taghizadeh, A. Hadizadeh, and L. Dejam, "Effect of Thickness and Annealing on Structural and Optical Properties of Bi2Te3 Thin Films Prepared from Bi2Te3 Nanoparticels," International Journal of Thin Films Science and Technology, vol. 3, no. 1, pp. 13, 2014. http://dx.doi.org/10.12785/ijtfst/030103.

[79] X. H. Yang, X. Y. Qin, J. Zhang, D. Li, H. X. Xin, and M. Liu, "Enhanced thermopower and energy filtering effect from synergetic scattering at heterojunction potentials in the thermoelectric composites with semiconducting nanoinclusions," Journal of Alloys and Compounds, vol. 558, pp. 203-211, 2013. https://dx.doi.org/10.1016/j.jallcom.2012.12.048.

[80] T. H. Zou, X. Y. Qin, D. Li, G. L. Sun, Y. C. Dou, Q. Q. Wang, B. J. Ren, J. Zhang, H. X. Xin, and Y. Y. Li, "Simultaneous enhancement in thermoelectric power factor and phonon blocking in hierarchical nanostructured $\beta$-Zn4Sb3-Cu3SbSe4," Applied Physics Letters, vol. 104, no. 1, pp. 13904, 2014. https://doi.org/10.1063/1.4861156.

[81] M. Elsheikh, D. Shnawah, M. Sabri, S. Said, M. Hassan, M. Bashir, and M. Mohamad, "A review on thermoelectric renewable energy: Principle parameters that affect their performance," Renewable \& Sustainable Energy Reviews, vol. 30, pp. 337-355, 2014. https://doi.org/10.1016/j.rser.2013.10.027. 